\title{
Genetic and Environmental Influences on Self- Reported Cognitive Functioning: Associations of Diverse Measures of Stress across the FMR1 CGG Repeat Range
}

Nell Maltman ( $\square$ anmaltman@wisc.edu )

University of Wisconsin Madison Waisman Center https://orcid.org/0000-0001-7874-9925

\section{Leann Smith DaWalt}

University of Wisconsin Madison Waisman Center

Jinkuk Hong

University of Wisconsin Madison Waisman Center

Mei Wang Baker

Wisconsin State Laboratory of Hygiene

Elizabeth M Berry-Kravis

Rush University Medical Center

Murray H. Brilliant

University of Wisconsin Madison Waisman Center

Marsha Mailick

University of Wisconsin Madison Waisman Center

\section{Research}

Keywords: Stress, Cognitive Function, Executive Function, Memory, FMR1, CGG Repeats

Posted Date: July 21st, 2020

DOI: https://doi.org/10.21203/rs.3.rs-18291/v2

License: (c) (i) This work is licensed under a Creative Commons Attribution 4.0 International License.

Read Full License 


\section{Abstract}

Background: The FMR1 gene is essential for neural development and healthy synaptic function. The modal number of CGG repeats in FMR1 is 30 , but the range is large with the reported copy number extending down to as few as 6 CGGs and up to over 200 CGGs. Prior work suggests that behavioral phenotypes, including cognitive function, may vary along the continuum of the FMR1 CGG repeat range. Stress also negatively influences cognitive function; however, it is not known whether FMR1-related variability (i.e., CGG repeat length), in addition to stress, independently influences cognitive function across the CGG range.

Methods: Participants included 1275 mothers who had between 18 and 123 CGG repeats. Participants completed self-report measures of executive function (BRIEF-A), memory, subjective stress (i.e., perceived stress), and objective stress (i.e., number of life events, parenting a child with a disability). Stress and CGG repeat length were examined as predictors of self-reported executive function and memory difficulty.

Results: Each measure of stress (i.e., perceived stress, life events, and parenting a child with a disability) significantly predicted greater self-reported difficulties in executive function and the likelihood of memory problems, net of age and level of education. Additionally, above and beyond stress effects, CGG repeat number significantly predicted executive functioning and memory difficulties.

Conclusions: These findings suggest that CGG repeat length confers independent contributions to selfreported executive function difficulty and memory problems over and above indices of stress, suggesting additive effects of genetic variation and environmental exposure.

\section{Background}

Optimal functioning of the fragile $\mathrm{X}$ mental retardation 1 (FMR1) gene and expression of its protein product, FMRP, are essential for normal neurodevelopment and synaptic function, and FMRP-regulated mRNA translation at the synapse (1). An expansion of more than 200 cytosine-guanine-guanine (CGG) trinucleotide repeats in the $5^{\prime}$ untranslated region of the FMR1 mRNA causes the gene to be fully methylated, interferes with protein production, and results in the full mutation of fragile $\mathrm{X}$ syndrome (FXS). Prior research exploring genotype-phenotype associations has largely focused on variation at the upper end of the CGG repeat range (2-6). Only a small body of literature has investigated the importance of CGG repeats continuously across this range with respect to both biological (7-9) and behavioral (10, 11) phenotypes. Thus, consideration of the FMR 1 CGG repeat number continuously across the range below FXS has the potential to elucidate phenotypic variation at the population-level. Of additional importance is the examination of environmental circumstances that may influence phenotypic variation. Prior work suggests that stress, such as parenting a child with a disability, may uniquely influence health outcomes across the CGG range (10). The present study aimed to characterize environmental and genetic predictors of self-reported cognitive function, specifically executive function and memory, across the CGG repeat range. 


\section{Cognitive Function and FMR1-related Variability}

Executive function and memory encompass higher-order mental processes required for goal-directed behavior (e.g., planning, inhibition, performance monitoring) and retrieval (12). Although difficulties in these domains have been observed in individuals who have CGG expansions $(3,4,13)$, there remains some controversy regarding the extent to which variation in these phenotypes may be directly the result of the repeat expansions, or alternatively may be explained by ascertainment bias, namely the inclusion in research of individuals with CGG expansions who also have children with FXS (14-16). To better tease apart these effects, one potentially fruitful approach is to examine the FMR1 CGG range (normal through premutation expansions) in relationship to variation in executive function and memory. Among the few studies to have done so, Hunter and colleagues (16) examined whether FMR1 CGG repeat length predicted factor scores reflecting aspects of executive function and memory. Among women, greater CGG repeat length was predictive of higher levels of self-reported inattention and impulsivity, as well as directassessment processing speed, but did not predict other factors (e.g., memory, response fluency). Though these findings were interpreted as marginal after correcting for multiple comparisons, this and several clinical reports (17-19) set the stage for continued exploration of executive function variability along the CGG repeat continuum. Building on this prior work, the present study examines cognitive functioning in individuals across the CGG repeat range below the full mutation.

\section{Stress and Cognitive Function}

Heightened levels of stress, both subjective and objective, have been reported in observational studies to be associated with executive dysfunction across multiple populations, including individuals with disabilities, individuals exposed to trauma, older adults, and healthy adult controls (12, 20-25). Greater perceived stress, a measure of subjective stress in response to external stressors, has been found to be associated with poorer cognitive functioning including attentional control, processing speed, and working memory $(20,22)$. Likewise, objective indices of stress (e.g., life events, parenting a child with a disability) have been associated with similar aberrations in executive function across diverse populations $(24,25)$.

These objective stressors have been found to be associated with cognitive dysfunction (including poorer episodic memory) (26), decreased well-being (27), poor mental health $(28,29)$, as well as changes to biological mechanisms, such as dampened cortisol-awakening-responses (30-32), and alterations to the neural structures that underlie stress-related responses $(21,22,33)$.

Parenting stress, or adverse psychological responses to parenting obligations (34), may be observed at increased rates in parents of children with disabilities due to unique and chronic caregiving demands. Meta-analyses $(35,36)$ suggest that parents of a child with a developmental disability experience higher rates of parenting stress than parents of typically developing children. Parenting stress has also been observed at increased rates in parents of children with other health conditions. For instance, having a child with a chronic physical (e.g., epilepsy) or mental health (e.g., bipolar disorder) condition has been associated with higher parenting stress compared to the parenting stress of raising typically developing children (37-39). Parents of adult children with developmental or mental health conditions have been 
exposed to this unique stressor for many years (28) and have been shown to have particularly high levels of parenting stress.

Past research has suggested that mothers may be more negatively affected by parenting stress than fathers (40-42). Therefore, the present study focused on mothers and examined the effects of multiple indices of stress on executive function and memory in mothers across the CGG repeat range (below the full mutation). In addition to stress, it is possible that other factors may contribute to variability in executive function and memory, namely age and education (43-45). These individual factors are therefore incorporated into the study as covariates to account for sociodemographic features that may influence variation in cognitive function. We hypothesized that subjective and objective stressors, as well as increased FMR1 CGG repeat number, would each independently predict executive function and memory difficulties, net of age and education.

\section{Methods}

\section{Participants and Procedures}

The sampling plan for this research was designed to include a sufficient number of participants from the full CGG repeat range to evaluate genotype-phenotype associations. The number of CGG repeats in FMR1 in the human population is not evenly distributed across the CGG range and is highly polymorphic (46, 47). The peak value for CGG repeats is 30 , with $>90 \%$ of individuals having fewer than 40 repeats and the lowest number of repeats ever reported being $6(7,46,48,49)$.

Four categories along the CGG repeat range (below FXS) have been described in the literature: premutation, intermediate zone, normal, and low zone $(9,50-54)$. However, precisely defining the number of repeats in each category is challenging due to both scientific and technical factors. The published guidelines provided by the American College of Medical Genetics and Genomics for defining normal and mutation categories in FMR1 (52) note that the borders of the various categories are approximate. "Each definition may change with increased empirical data and research" (p. 578), and there is an acceptable margin of error of several CGG repeats at the borders of the categories. For this reason, in the current research, we investigate the phenotypic associations of variation in CGG repeats by treating repeat number as a continuous variable.

Participants included 1275 mothers with CGG repeats ranging from 18 to 123.

The majority of these participants $(n=1152)$ were drawn from the Marshfield Clinic Personalized Medicine Research Project (PMRP) (55), a 20,000-person population-based biobank. Individuals enrolled in this biobank in the early 2000s, and provided written informed consent to provide researchers with access to their DNA and electronic health records, and to be contacted for additional data collection. Per IRB, research results were not returned to participants, nor were the results entered into their medical record or provided to health care personnel. Over half of the PMRP members were female $(n=11,556)$ and DNA was available for $99.7 \%$ of them. 
For a previous investigation (56), the DNA samples of all PMRP members were screened for FMR1 CGG repeats. This screening made it possible to select participants for the present study from across the CGG repeat range, with adequate numbers of individuals at the lower and higher ends of the range. Based on previous research defining the number of CGG repeats that can be considered expansions (53,57-61), we invited all PMRP females who had at least 41 CGG repeats on one allele to participate in the present study. Similarly, we invited all those who had at least one allele below the normal range (defined here as below 26 CGG repeats; $(50,54)$ to participate. Additionally, based on a power analysis, a random sample of females with normal-range CGGs was selected for inclusion in the present research. Thus, by design, the recruited sample included all females in the population biobank who had expanded or low numbers of CGGs, and a random sample of females in the normal range. The response rate of the recruited females was $77.4 \%$. We further restricted the current analysis to data obtained from mothers who had at least one biological or adopted child.

The CGGs of the participants from PMRP ranged from 18 - 100 repeats. Of note, 44 of these participants had CGG repeats in the premutation range (55+ CGG repeats). To extend the range of FMR1 CGG repeats, clinically-ascertained mothers of children diagnosed with FXS were included in the present analysis $(\mathrm{n}=$ 123, with 67-123 CGGs). Participants from the clinically-ascertained samples were recruited from fragile $X$ clinics, via local media, newsletters, brochures, and disability registries $(62,63)$. All participants (PMRP and clinically-ascertained) completed a questionnaire that provided information on whether they had a child with a developmental or mental health condition (see Table 1), as well as all other non-genetic measures for the current study.

The Institutional Review Boards at the University of Wisconsin-Madison and the Marshfield Clinic approved all procedures and all participants signed informed consents.

\section{Measures}

\section{Stress.}

Perceived Stress Scale. The Perceived Stress Scale (PSS) (64) is a 10-item, self-report measure that quantifies an individual's appraisal of stressful experiences from the past month. Examples include "In the last month, how often have you felt difficulties were piling up so high that you could not overcome them?" and "In the last month how often did you feel nervous or stressed?" Each item was scored on a scale of 0 (never) - 4 (very often); four positively-stated items were reverse-coded. The total score represents a sum across items; higher numbers indicate a greater degree of perceived stress (i.e., subjective stress). Age- and gender-based norms were previously established ( $\mathrm{n}=1406$ females; $M=$ $13.7, S D=6.6)$ with a Cronbach $a$ coefficient of $.78(65)$. Higher PSS scores have been linked to poorer health including greater risk of developing depressive symptoms following life events and vulnerability to the common cold (65).

Life Events. Participants reported life events (positive and negative) that they personally experienced during the past year (adapted from Abidin's Parenting Stress Index; (66). Participants selected events 
from a list of 22 items, such as divorce, going into debt, and the birth of a child. Higher scores indicate a greater number of personal life events.

Parenting Status. Participants reported whether their child had a developmental or mental health condition ( 0 = no, $1=$ yes $)$.

\section{Cognitive function.}

Executive Function. Participants completed the Behavior Rating Inventory of Executive Function-Adult Version (BRIEF-A) $(67,68)$, a well-validated self-report measure of executive function in daily life for adults. The BRIEF-A consists of 75-items that yields an overall raw score of executive function (Global Executive Composite; GEC), made up of two indices: Behavior Regulation Index (BRI) and Metacognitive Index (MI). Participants indicated the extent to which they experienced problems across nine domains: Inhibit, Shift, Emotional Control, Self-Monitor, Initiate, Working Memory, Plan/Organize, Task Monitor, and Organization of Materials, which together comprise the GEC (see Table 2 for definitions). Each item was rated from 1 (never) to 3 (often). Raw scores for each domain were converted into t-scores, with higher scores suggestive of greater executive difficulties in daily life. T-scores that exceeded 65 on any domain indicated clinically-significant executive dysfunction in that area. In order to ensure that respondents did not indicate excessively negative self-perception about their own executive function, the Negativity scale was examined to ensure that no participant met or exceeded a total score of six (67).

The BRIEF-A was previously standardized on a representative population sample of 1136 adults with Cronbach $a$ coefficients ranging from $.93-.96$ and test-retest reliability ranging from $.93-.94$ across domains, with utility demonstrated in both clinical and non-clinical samples (67-70). The BRIEF-A has been shown to correlate significantly with direct-assessment measures of executive function (e.g., go/no go and trail making tests) in healthy adults (71) and in individuals with disorders associated with executive dysfunction (72-74). The present study used the GEC $t$-score as the indicator of executive functioning.

The BRIEF-A was standardized on participants ages 18-90. Since there were 12 participants in the present sample who were over the age of 90 , we checked all findings excluding participants over age 90 , which did not change results. Therefore, the findings reported below include all participants.

Self-reported Memory Problems. Participants answered the question: Do you have problems with memory? This question was rated as 0 (no problems with memory), 1 (undiagnosed problems with memory), or 2 (diagnosed memory problems). Only 20 mothers ( $1.6 \%$ of the sample) reported diagnosed memory problems. To reduce skewness, all "2" responses were collapsed to "1".

FMR1-related variation. DNA samples were obtained from cheek swabs and blood samples from all participants, and were analyzed for CGG repeats in FMR1. Assays were completed at the Wisconsin State Laboratory of Hygiene and the Rush University Medical Center Molecular Diagnostics Laboratory, using 
procedures described previously $(56,63,75,76)$. Mothers who were mosaic for the full mutation were excluded from the present analyses.

The DNA of the clinically-ascertained mothers of children with FXS was obtained from cheek swabs, and CGG repeat length was assayed in the laboratory of Elizabeth Berry-Kravis, MD, PhD at Rush University. The DNA of the PMRP members was obtained from blood samples, tested either in the Rush laboratory or in the Wisconsin State Laboratory of Hygiene under the supervision of Mei Wang Baker, MD.

\section{Statistical Analyses}

Statistical analyses were performed using IBM SPSS Statistics, version 26 (77). Descriptive statistics and Pearson correlations among all study variables are presented in Table 3. Maternal age and education were controlled in all subsequent analyses. To control for potential effects of the second FMR1 allele (as females carry two X chromosomes), the "shorter" allele (i.e., the allele with the lower number of CGG repeats) was included as a covariate in all regression analyses.

For the domain of executive function, the primary analysis involved three hierarchical regressions (one for each type of stressor) that assessed the key prediction that stress and FMR1 CGG repeat length would each uniquely contribute to self-reported executive function difficulties. For memory problems, three logistic regressions (one for each type of stressor) were completed to test if stress and FMR1 CGG repeat length would predict the likelihood of a self-reported memory problem. For both executive function and memory problem models, maternal age, education, the number of CGG repeats on the shorter allele, and each stress measure were entered into the first block; CGG repeat length (on the long allele) was entered into the second block. In the third block, a separate term (CGG squared) was included in all regression models to evaluate potential curvilinear CGG effects within the sample. A significant curvilinear effect would suggest that components of the CGG distribution (e.g., premutation expansions) would potentially be driving the CGG effect, whereas if the curvilinear effect is not significant, this would suggest that the CGG effect is linear across the full CGG range. Following the approach of Hunter et al. (16), a Bonferonni correction was used to adjust for multiple testing for each indicator of cognitive functioning, with the alpha level set at $p=.016(0.05 / 3)$.

\section{Results}

\section{Descriptive Findings}

Participants' ages ranged from $28-98$ years $(M=57.83, S D=14.90)$. Almost all mothers self-identified as White $(99.2 \%)$. The majority of the mothers $(62.3 \%)$ had graduated from college. Their children ranged in age from $<1$ year to 71 years $(M=30.30, S D=13.81)$. The number of children in each family ranged from 1 to $13(M=2.79, S D=1.50)$; the number of children in each family with a developmental or mental health condition ranged from 0 to $6(M=.38, S D=.78)$. Fully $43.5 \%$ of the mothers had a child with a developmental or mental health condition. Perceived stress ranged from 0 to $39(M=13.22, S D=7.39)$, similar to the normative population. Most participants (62.2\%) had experienced at least one life event in 
the past year $(M=1.25, S D=1.42$, Range $=0$ - 9). GEC t-scores on the BRIEF-A ranged from 35 to $93(M=$ $50.44, S D=10.60)$, again similar to the normative population. Only $11 \%$ of participants exceeded clinical cutoff on the GEC (i.e., t-score > 65). Approximately $25 \%$ of participants self-reported a memory problem.

Correlations among study variables are depicted in Table 3. Notably, the stress measures were significantly inter-correlated ( $p$-values $<.001)$, although the strengths of these associations were small to moderate $(r s=.142-.322)$, indicating that the three measures represented somewhat distinct aspects of stress. Both executive function difficulty and memory problems were significantly associated with each measure of stress and CGG repeat length ( $p$-values $<.001)$. Executive function difficulty and memory problems were significantly correlated with each other, with moderate effects $(r=.355 ; p<.001)$.

\section{Multivariate Findings}

The key predictions of the study were that stress would influence executive function and memory function, and that an effect of FMR1 CGG repeat length would be associated with cognitive function above and beyond indices of stress.

Executive Function. Curvilinear CGG effects were not significant in any model of executive function ( $p$ values $>.589$ ); thus, linear effects are reported below. For the full models showing the non-significant curvilinear coefficients, see Supplemental Materials.

As presented in Table 4, perceived stress (PSS) significantly predicted variance in the global executive composite (GEC) of the BRIEF-A ( $b=.866, p<.001)$, such that higher levels of perceived stress were associated with greater problems in executive functioning. There was an additional significant effect of CGG repeat length ( $b=.036, p=.011$; see Figure 1$)$. Similarly, total number of life events significantly predicted higher executive functioning problems $(b=1.786, p<.001)$ with additional significant CGG effects ( $b=.073, p<.001$; see Figure 2$)$. Finally, higher executive functioning problems were significantly predicted by parenting a child with a developmental or mental health condition $(b=2.869, p<.001)$ and by greater CGG repeat length ( $b=.045, p=.012$; see Figure 3 ). All tests of the CGG repeat length survived correction for multiple testing.

Estimated regression lines indicate the independent influences of CGG repeat length and increased number of life events on executive function difficulty. Trend lines represent a subset of participants with high life events ( $>1$ SD above the mean) and low life events ( $<1$ SD below the mean).

Estimated regression lines indicate the independent influences of CGG repeat length and parenting a child with a disability on executive function difficulty. 
Memory Problems. Second, we predicted that stress and FMR1 CGG repeat length would independently predict the likelihood of self-reported memory problems. The curvilinear term was tested and found to be significant in all models reported below. However, the significant CGG repeat effects in these models did not survive correction for multiple testing, and thus are interpreted as marginal. For the full models showing the curvilinear coefficients, see Supplemental Materials.

As shown in Table 5, PSS significantly predicted self-reported memory problems $(\mathrm{OR}=1.085, p<.001)$, with additional significant $C G G$ effects $(O R=1.017, p<.001$; see Figure 4). Total number of life events significantly predicted self-reported memory problems $(\mathrm{OR}=1.229, p<.001)$, with additional significant CGG effects (OR $=1.020, p<.001$; see Figure 5$)$. Last, self-reported memory problems were predicted by parenting status $(\mathrm{OR}=1.793, p<.001)$, with additional significant $\mathrm{CGG}$ effects $(\mathrm{OR}=1.015, p<.001$; see Figure 6). All tests of the CGG repeat length survived correction for multiple testing.

Covariates. Both age and education were significant predictors of executive function difficulty across all models ( $p$-values $\leq .022$ ). Age and education were significant predictors of self-reported memory problems in all models that included CGG repeat length ( $p$-values $\leq .034)$.

\section{Discussion}

The present study evaluated the influence of distinct dimensions of stress, and the independent effects of FMR1 CGG repeat length (up to but excluding the full mutation), on self-reported cognitive functioning (i.e., executive function and memory). Importantly, CGG repeat length accounted for small, but statistically significant elevations in executive function difficulty and memory problems, above and beyond stress, age and level of education. To date, this study represents the largest sample in which the association between cognitive function and FMR1 CGG repeat length has been studied. By taking a continuous approach to evaluating FMR7-related effects on cognitive function, and by assessing mothers of non-disabled children as well as children with a diverse range of disability conditions, this study advances understanding of how both environmental and genetic factors influence self-reported cognitive functioning at the population level.

Historically, examination of behavioral phenotypes associated with FMR1-related variability (e.g., CGG repeat length) have largely focused on individuals with full mutation fragile $X$ syndrome or the premutation, with some exceptions $(10,11,16,78)$. Many prior assessments of cognition associated with the FMR1 gene involved group comparisons, typically between PM carriers and those with modal numbers of CGG repeats $(2,4,5,16)$. With consideration of the continuous nature of the CGG repeat range, as in the present study, the interpretation of the relationship between FMR1-related variation and phenotypic expression can be advanced.

Our initial findings revealed linear effects of CGG repeat length on executive functioning and curvilinear effects of CGG repeat length on memory problems. Higher incidence of memory problems was evident at 
approximately $80 \mathrm{CGG}$ repeats, suggesting that repeats in the premutation range may be driving this effect. However, the curvilinear effect of CGG repeats for the prediction of memory problems did not survive correction for multiple testing, and were thus at a trend level.

Limitations in executive functioning and memory problems may represent distinct aspects of cognition. Higher scores on the BRIEF-A reflect difficulty sitting still and waiting, the propensity to make untactful remarks, and the tendency to complete tasks in a hurried manner. Endorsement of the item reflecting memory problems reflects the self-perception that one has difficulties with everyday memory. Thus, the present results suggest that CGG repeat number across the full range is predictive of a broad range of cognitive difficulties above and beyond demographic factors (age and education) and several types of stress. Replication of the current findings is necessary, and research examining the basic biological functions of the FMR1 CGG repeat is needed to fully understand these effects.

Although the primary focus of the present study was the effect of CGG repeats on cognitive functioning, the results also suggest that each specific type of stress provided unique insights about stress effects on cognitive functioning. For example, the life events that were experienced during the past year encompassed a variety of events that are not necessarily "negative", such as the birth of a child, increased income, and moving to a new home. Luhmann and colleagues (27) conducted a meta-analysis on relationships between life events and subjective well-being, including cognitive well-being. Similar to our study, they found that cognitive well-being varied in response to the presence of life events, both positive and negative, which may simply be an indication that life change is stressful and can affect cognition.

Though parenting status was a significant predictor of higher self-reported problems with executive function and memory, it predicted less variance than perceived stress and life events. The conditions of the children represented in the sample varied considerably. Whereas some mothers had children with developmental conditions typically present at birth or in early childhood (e.g., Down syndrome), other conditions are later in onset (e.g., schizophrenia). Furthermore, while some conditions were rare, most conditions were relatively common, as the majority of affected children had conditions such as anxiety, depression, or ADHD. The differences in duration and severity of stress exposure to these varied conditions may have influenced the magnitude of variance in cognitive function predicted by parenting a child with a developmental or mental health condition. These factors warrant further attention in future work.

Additionally, age and education each significantly contributed to variance in cognitive function in addition to stress and CGG repeat effects, as suggested in prior research $(43,44)$. Prior work shows that agerelated cognitive problems are most pronounced for individuals with lower levels of education (45). The present research suggests that studies of the relationships between variation in the FMR1 CGG repeat number and behavioral phenotypes should consider additional individual and environmental factors to accurately evaluate FMRT-related influences.

\section{Study Strengths, Limitations, and Future Directions}


This study had several notable strengths. First, the availability of DNA and FMR1 CGG repeat assays across the full range of CGG repeats (below the full mutation) enabled robust examination of the effects of FMR1 repeat-related variability on self-reported cognitive function. Second, we had a large sample size, which drew primarily from a population-based biobank. Third, this study was strengthened by consideration of multiple measures of stress, providing a robust test of the overall hypothesis. Additionally, the inclusion of child disabilities was broad, further contributing to the generalizability of study findings.

This study also had some limitations. Although the sample was diverse with regards to age and the range of FMR1 repeat-related variation, the participants in the sample were racially and ethnically homogenous. Additionally, many prior reports of associations between cognitive function and FMR1 CGG expansions have included direct-assessment measures, whereas the present study relied on self-report. The study's large sample size precluded direct testing of $>1200$ individuals. It has been suggested that PM carriers may over-report symptoms not evidenced on neurological exam (79). However, there is also extant literature confirming significant associations between cognitive function and CGG repeat length using both direct-assessment and self-report measures across the CGG range $(16,80)$, suggesting the validity of self-reported results. Hunter and colleagues (16) have discussed the possibility that individuals who participate in research may be less likely to have cognitive difficulties. Thus, while it is possible that the present findings could reflect potential ascertainment bias in recruited individuals (who are less likely to experience cognitive difficulties), the high response rate from our sample recruitment (77.4\%) is indicative of a sample that is largely representative of the population from which participants were drawn.

Another limitation of the present study is that the only FMR1-related biomarker available for the study participants was CGG repeat number. Inclusion of activation ratio, mRNA, and FMRP levels would greatly enhance understanding of the processes investigated here. Additionally, interpretation of these findings can only be extrapolated to females. Studies of males across the CGG range would clarify generalizability of these findings. Finally, the participants in the present study were recruited using multiple methods, including drawing from a 20,000-person population-based biobank and via a national sample of premutation carriers who were identified clinically after a child was diagnosed with FXS. Although this approach made it possible to include participants with repeats ranging from 18 to 123 CGGs, in future research, it would be advantageous to use a single method of recruitment across diverse samples, but that would require access to much larger population biobanks.

\section{Conclusions}

Findings from the present study highlight the importance of separately considering the role of stress and FMR1-related variability in studies of cognitive function. Both stress and CGG repeat length independently predicted variation in self-reported executive function and the likelihood of memory problems. Future work should incorporate multiple dimensions of FMR1-related biomarkers and objective cognitive testing to advance understanding of genotype-phenotype associations at the population level. 


\section{Declarations}

\section{Ethics approval and consent to participate}

The Institutional Review Boards at the University of Wisconsin-Madison and the Marshfield Clinic approved all procedures and all participants signed informed consents prior to participation. Relevant IRB protocols from the Marshfield Clinic Research Institute were: IRB-18-225, IRB-18-157, IRB-18-382. The relevant IRB protocol from UW-Madison was 2013-0510.

\section{Consent for publication}

Consent for publication is not applicable.

\section{Availability of data and materials}

The datasets generated for this study will not be made publicly available. The terms of the IRB protocols prohibit public sharing of the data sets.

\section{Competing interests}

MM is the Chair of the Scientific Advisory Board of the John Merck Fund.

EBK has received funding from Seaside Therapeutics, Novartis, Roche, Alcobra, Neuren, Cydan, Fulcrum, GW, Neurotrope, Marinus, Zynerba, BioMarin, Ovid, Tetra, Retrophin, Yamo, Acadia, Avexis, Ionis, Ultragenyx, Lumos, GeneTx, and Vtesse/Sucampo/Mallinkcrodt Pharmaceuticals to consult on trial design or development strategies and/or conduct clinical trials in FXS or other NDDs or neurodegenerative disorders, and from Asuragen Inc to develop testing standards for FMR1 testing. All funding to EBK is directed to Rush University Medical Center to support rare disease programs. EBK receives no personal funds.

The remaining authors declare they have no competing interests.

\section{Funding}

Research reported in this publication was supported by the Eunice Kennedy Shriver National Institute of Child Health \& Human Development of the National Institutes of Health under Award Numbers R01 HD082110 (PI: MM), T32 HD007489, and U54 HD090256 and the Waisman Center at the University of Wisconsin-Madison.

\section{Authors' contributions}

MM, LSD, and JH designed the larger study from which the data were derived and supervised the data collection. NM and $\mathrm{JH}$ analyzed the data. NM, LSD, and MM wrote the first draft and made edits to the manuscript. MHB contributed the DNA samples and facilitated the collection of data from the 
participants in Marshfield PMRP. MWB and EB-K conducted the CGG repeat assays. All authors contributed to interpretation of the data and approved the final manuscript.

\section{Acknowledgements}

We would like to acknowledge all of the families who participated in this study. We would also like to acknowledge Jan Greenberg for his contributions to the study and comments on the manuscript.

\section{Abbreviations}

BRIEF-A: Behavior rating inventory of executive function-adult version

FMR1: Fragile $\mathrm{X}$ mental retardation 1

FXS: Fragile X syndrome

PM: Premutation

PSS: Perceived Stress Scale

\section{References}

1. Darnell JC, Van Driesche SJ, Zhang C, Hung KY, Mele A, Fraser CE, et al. FMRP stalls ribosomal translocation on mRNAs linked to synaptic function and autism. Cell. 2011;146(2):247-61.

2. Shelton AL, Cornish K, Fielding J. Long term verbal memory recall deficits in fragile $\mathrm{X}$ premutation females. Neurobiol Learn Mem. 2017;144:131-5.

3. Shelton AL, Cornish KM, Godler DE, Clough M, Kraan C, Bui M, et al. Delineation of the working memory profile in female FMR1 premutation carriers: the effect of cognitive load on ocular motor responses. Behav Brain Res. 2015;282:194-200.

4. Shelton AL, Cornish KM, Kraan CM, Lozano R, Bui M, Fielding J. Executive Dysfunction in Female FMR1 Premutation Carriers. Cerebellum. 2016;15(5):565-9.

5. Hippolyte L, Battistella G, Perrin AG, Fornari E, Cornish KM, Beckmann JS, et al. Investigation of memory, executive functions, and anatomic correlates in asymptomatic FMR1 premutation carriers. Neurobiol Aging. 2014;35(8):1939-46.

6. Birch RC, Hocking DR, Trollor JN. Prevalence and predictors of subjective memory complaints in adult male carriers of the FMR1 premutation. Clin Neuropsychol. 2016;30(6):834-48.

7. Chen LS, Tassone F, Sahota P, Hagerman PJ. The (CGG)n repeat element within the $5^{\prime}$ untranslated region of the FMR 1 message provides both positive and negative cis effects on in vivo translation of a downstream reporter. Hum Mol Genet. 2003;12(23):3067-74.

8. Kenneson A, Zhang F, Hagedorn CH, Warren ST. Reduced FMRP and increased FMR1 transcription is proportionally associated with CGG repeat number in intermediate-length and premutation carriers. 
Hum Mol Genet. 2001;10(14):1449-54.

9. Loesch DZ, Bui QM, Huggins RM, Mitchell RJ, Hagerman RJ, Tassone F. Transcript levels of the intermediate size or grey zone fragile $X$ mental retardation 1 alleles are raised, and correlate with the number of CGG repeats. J Med Genet. 2007;44(3):200-4.

10. Mailick M, Hong J, Greenberg J, Dawalt LS, Baker MW, Rathouz PJ. FMR1 genotype interacts with parenting stress to shape health and functional abilities in older age. Am J Med Genet B Neuropsychiatr Genet. 2017;174(4):399-412.

11. Klusek J, Porter A, Abbeduto L, Adayev T, Tassone F, Mailick MR, et al. Curvilinear Association Between Language Disfluency and FMR1 CGG Repeat Size Across the Normal, Intermediate, and Premutation Range. Front Genet. 2018;9:344.

12. Diamond A. Executive functions. Annu Rev Psychol. 2013;64:135-68.

13. Grigsby J, Cornish K, Hocking D, Kraan C, Olichney JM, Rivera SM, et al. The cognitive neuropsychological phenotype of carriers of the FMR1 premutation. J Neurodev Disord. 2014;6(1):28.

14. Gossett A, Sansone S, Schneider A, Johnston C, Hagerman R, Tassone F, et al. Psychiatric disorders among women with the fragile $X$ premutation without children affected by fragile $X$ syndrome. Am $J$ Med Genet B Neuropsychiatr Genet. 2016;171(8):1139-47.

15. Wheeler AC, Bailey DB, Berry-Kravis E, Greenberg J, Losh M, Mailick M, et al. Associated features in females with an FMR1 premutation. J Neurodev Disord. 2014;6(1):30.

16. Hunter JE, Allen EG, Abramowitz A, Rusin M, Leslie M, Novak G, et al. No evidence for a difference in neuropsychological profile among carriers and noncarriers of the FMR1 premutation in adults under the age of 50. Am J Hum Genet. 2008;83(6):692-702.

17. Debrey SM, Leehey MA, Klepitskaya O, Filley CM, Shah RC, Kluger B, et al. Clinical Phenotype of Adult Fragile X Gray Zone Allele Carriers: a Case Series. Cerebellum. 2016;15(5):623-31.

18. Hall DA, Berry-Kravis E, Zhang W, Tassone F, Spector E, Zerbe G, et al. FMR1 gray-zone alleles: association with Parkinson's disease in women? Mov Disord. 2011;26(10):1900-6.

19. Hall DA, Nag S, Ouyang B, Bennett DA, Liu Y, Ali A, et al. Fragile X Gray Zone Alleles Are Associated With Signs of Parkinsonism and Earlier Death. Mov Disord. 2020.

20. Liston C, McEwen BS, Casey BJ. Psychosocial stress reversibly disrupts prefrontal processing and attentional control. Proc Natl Acad Sci U S A. 2009;106(3):912-7.

21. Lupien SJ, McEwen BS, Gunnar MR, Heim C. Effects of stress throughout the lifespan on the brain, behaviour and cognition. Nat Rev Neurosci. 2009;10(6):434-45.

22. Korten NC, Comijs HC, Penninx BW, Deeg DJ. Perceived stress and cognitive function in older adults: which aspect of perceived stress is important? Int J Geriatr Psychiatry. 2017;32(4):439-45.

23. Shields GS, Sazma MA, Yonelinas AP. The effects of acute stress on core executive functions: A meta-analysis and comparison with cortisol. Neurosci Biobehav Rev. 2016;68:651-68. 
24. Heyman M, Hauser-Cram P. Negative life events predict performance on an executive function task in young adults with developmental disabilities. J Intellect Disabil Res. 2015;59(8):746-54.

25. Op den Kelder R, Ensink JBM, Overbeek G, Maric M, Lindauer RJL. Executive function as a mediator in the link between single or complex trauma and posttraumatic stress in children and adolescents. Qual Life Res. 2017;26(7):1687-96.

26. Song J, Mailick MR, Greenberg JS, Ryff CD, Lachman ME. Cognitive Aging in Parents of Children with Disabilities. J Gerontol B Psychol Sci Soc Sci. 2016;71(5):821-30.

27. Luhmann M, Hofmann W, Eid M, Lucas RE. Subjective well-being and adaptation to life events: a meta-analysis. J Pers Soc Psychol. 2012;102(3):592-615.

28. Seltzer MM, Floyd F, Song J, Greenberg J, Hong J. Midlife and aging parents of adults with intellectual and developmental disabilities: impacts of lifelong parenting. Am J Intellect Dev Disabil. 2011;116(6):479-99.

29. Barker ET, Hartley SL, Seltzer MM, Floyd FJ, Greenberg JS, Orsmond GI. Trajectories of emotional well-being in mothers of adolescents and adults with autism. Dev Psychol. 2011;47(2):551-61.

30. Seltzer MM, Barker ET, Greenberg JS, Hong J, Coe C, Almeida D. Differential sensitivity to life stress in FMR1 premutation carrier mothers of children with fragile $X$ syndrome. Health Psychol. 2012;31(5):612-22.

31. Barker ET, Greenberg JS, Seltzer MM, Almeida DM. Daily stress and cortisol patterns in parents of adult children with a serious mental illness. Health Psychol. 2012;31(1):130-4.

32. Harris MA, Cox SR, Brett CE, Deary IJ, MacLullich AM. Stress in childhood, adolescence and early adulthood, and cortisol levels in older age. Stress. 2017;20(2):140-8.

33. Piccolo LR, Noble KG. Perceived stress is associated with smaller hippocampal volume in adolescence. Psychophysiology. 2018;55(5).

34. Deater-Deckard K. Parenting Stress and Child Adjustment: Some Old Hypotheses and New Questions. Clinical Psychology: Science and Practice. 1998;5(3):314-32.

35. Hayes SA, Watson SL. The impact of parenting stress: a meta-analysis of studies comparing the experience of parenting stress in parents of children with and without autism spectrum disorder. $J$ Autism Dev Disord. 2013;43(3):629-42.

36. Barroso NE, Mendez L, Graziano PA, Bagner DM. Parenting Stress through the Lens of Different Clinical Groups: a Systematic Review \& Meta-Analysis. J Abnorm Child Psychol. 2018;46(3):449-61.

37. Cousino MK, Hazen RA. Parenting stress among caregivers of children with chronic illness: a systematic review. J Pediatr Psychol. 2013;38(8):809-28.

38. Farrace D, Tommasi M, Casadio C, Verrotti A. Parenting stress evaluation and behavioral syndromes in a group of pediatric patients with epilepsy. Epilepsy Behav. 2013;29(1):222-7.

39. Perez Algorta G, MacPherson HA, Youngstrom EA, Belt CC, Arnold LE, Frazier TW, et al. Parenting Stress Among Caregivers of Children With Bipolar Spectrum Disorders. J Clin Child Adolesc Psychol. 2018;47(sup1):S306-S20. 
40. Skreden M, Skari H, Malt UF, Pripp AH, Bjork MD, Faugli A, et al. Parenting stress and emotional wellbeing in mothers and fathers of preschool children. Scand J Public Health. 2012;40(7):596-604.

41. Pelchat $D$, Lefebvre $H$, Levert MJ. Gender differences and similarities in the experience of parenting a child with a health problem: current state of knowledge. J Child Health Care. 2007;11(2):112-31.

42. Pelchat D, Lefebvre H, Perreault M. Differences and similarities between mothers' and fathers' experiences of parenting a child with a disability. J Child Health Care. 2003;7(4):231-47.

43. Fjell AM, Sneve MH, Grydeland H, Storsve AB, Walhovd KB. The Disconnected Brain and Executive Function Decline in Aging. Cereb Cortex. 2017;27(3):2303-17.

44. Harada CN, Natelson Love MC, Triebel KL. Normal cognitive aging. Clin Geriatr Med. 2013;29(4):73752.

45. Taylor A, Bouldin ED, McGuire LC. Subjective cognitive decline among adults aged $\geq 45$ years - United States, 2015-2016. Morbidity and Mortality Weekly Report. 2018;67:753-7.

46. Fu YH, Kuhl DP, Pizzuti A, Pieretti M, Sutcliffe JS, Richards S, et al. Variation of the CGG repeat at the fragile X site results in genetic instability: resolution of the Sherman paradox. Cell. 1991;67(6):104758.

47. Eichler EE, Kunst CB, Lugenbeel KA, Ryder OA, Davison D, Warren ST, et al. Evolution of the cryptic FMR1 CGG repeat. Nat Genet. 1995;11(3):301-8.

48. Snow K, Doud LK, Hagerman R, Pergolizzi RG, Erster SH, Thibodeau SN. Analysis of a CGG sequence at the FMR-1 locus in fragile $X$ families and in the general population. Am J Hum Genet. 1993;53(6):1217-28.

49. Brown WT, Houck GE, Jr., Jeziorowska A, Levinson FN, Ding X, Dobkin C, et al. Rapid fragile X carrier screening and prenatal diagnosis using a nonradioactive PCR test. JAMA. 1993;270(13):1569-75.

50. Weghofer A, Tea MK, Barad DH, Kim A, Singer CF, Wagner K, et al. BRCA1/2 mutations appear embryo-lethal unless rescued by low (CGG $\mathrm{n}<26)$ FMR1 sub-genotypes: explanation for the "BRCA paradox"? PLoS One. 2012;7(9):e44753.

51. Maddalena A, Richards CS, McGinniss MJ, Brothman A, Desnick RJ, Grier RE, et al. Technical standards and guidelines for fragile $X$ : the first of a series of disease-specific supplements to the Standards and Guidelines for Clinical Genetics Laboratories of the American College of Medical Genetics. Quality Assurance Subcommittee of the Laboratory Practice Committee. Genet Med. 2001;3(3):200-5.

52. Monaghan KG, Lyon E, Spector EB, erican College of Medical G, Genomics. ACMG Standards and Guidelines for fragile $X$ testing: a revision to the disease-specific supplements to the Standards and Guidelines for Clinical Genetics Laboratories of the American College of Medical Genetics and Genomics. Genet Med. 2013;15(7):575-86.

53. Nolin SL, Lewis FA, Ye LL, Houck GE, Glicksman AE, Limprasert $P$, et al. Familial transmission of the FMR1 CGG repeat. Am J Hum Genet. 1996;59(6):1252-61.

54. Mailick MR, Hong J, DaWalt LS, Greenberg JS, Movaghar A, Baker MW, et al. FMR1 Low Zone CGG Repeats: Phenotypic Associations in the Context of Parenting Stress. Front Pediatr. 2020;8:223. 
55. McCarty CA, Wilke RA, Giampietro PF, Wesbrook SD, Caldwell MD. Marshfield Clinic Personalized Medicine Research Project (PMRP): design, methods and recruitment for a large population-based biobank. Per Med. 2005;2(1):49-79.

56. Maenner MJ, Baker MW, Broman KW, Tian J, Barnes JK, Atkins A, et al. FMR1 CGG expansions: prevalence and sex ratios. Am J Med Genet B Neuropsychiatr Genet. 2013;162B(5):466-73.

57. Bretherick KL, Fluker MR, Robinson WP. FMR1 repeat sizes in the gray zone and high end of the normal range are associated with premature ovarian failure. Hum Genet. 2005;117(4):376-82.

58. Hall D, Tassone F, Klepitskaya O, Leehey M. Fragile X-associated tremor ataxia syndrome in FMR1 gray zone allele carriers. Mov Disord. 2012;27(2):296-300.

59. Nolin SL, Brown WT, Glicksman A, Houck GE, Jr., Gargano AD, Sullivan A, et al. Expansion of the fragile X CGG repeat in females with premutation or intermediate alleles. Am J Hum Genet. 2003;72(2):454-64.

60. Hall DA. In the Gray Zone in the Fragile X Gene: What are the Key Unanswered Clinical and Biological Questions? Tremor Other Hyperkinet Mov (N Y). 2014;4:208.

61. Hall DA, Bennett DA, Filley CM, Shah RC, Kluger B, Ouyang B, et al. Fragile X gene expansions are not associated with dementia. Neurobiol Aging. 2014;35(11):2637-8.

62. Mailick MR, Hong J, Greenberg J, Smith L, Sherman S. Curvilinear association of CGG repeats and age at menopause in women with FMR1 premutation expansions. Am J Med Genet B Neuropsychiatr Genet. 2014;165B(8):705-11.

63. Mailick MR, Movaghar A, Hong J, Greenberg JS, DaWalt LS, Zhou L, et al. Health Profiles of Mosaic Versus Non-mosaic FMR1 Premutation Carrier Mothers of Children With Fragile X Syndrome. Front Genet. 2018;9:173.

64. Cohen S, Kamarck T, Mermelstein R. A global measure of perceived stress. J Health Soc Behav. 1983;24(4):385-96.

65. Cohen S, Williamson GM. Perceived stress in a probability sample in the United States. In: Spacapan S, Oskamp S, editors. The social psychology of health. Newbury Park, CA: Oxford; 1988. p. 31-67.

66. Abidin RR. Parenting stress index. 4th Edition ed. Lutz, FL: PAR; 2012.

67. Roth RM, Isquith PK, Gioia GA. Behavior rating inventory of executive function-adult version. Lutz, FL: Psychological Assessment Resources; 2005.

68. Roth RM, Lance CE, Isquith PK, Fischer AS, Giancola PR. Confirmatory factor analysis of the Behavior Rating Inventory of Executive Function-Adult version in healthy adults and application to attentiondeficit/hyperactivity disorder. Arch Clin Neuropsychol. 2013;28(5):425-34.

69. Christ SE, Kanne SM, Reiersen AM. Executive function in individuals with subthreshold autism traits. Neuropsychology. 2010;24(5):590-8.

70. Rabin LA, Roth RM, Isquith PK, Wishart HA, Nutter-Upham KE, Pare N, et al. Self- and informant reports of executive function on the BRIEF-A in $\mathrm{MCl}$ and older adults with cognitive complaints. Arch Clin Neuropsychol. 2006;21(7):721-32. 
71. Erkkila M, Perakyla J, Hartikainen KM. Executive Functions and Emotion-Attention Interaction in Assessment of Brain Health: Reliability of Repeated Testing With Executive RT Test and Correlation With BRIEF-A Questionnaire. Front Psychol. 2018;9:2556.

72. Rouel M, Raman J, Hay P, Smith E. Validation of the Behaviour Rating Inventory of Executive Function - Adult Version (BRIEF-A) in the obese with and without binge eating disorder. Eat Behav. 2016;23:58-65.

73. Grane VA, Endestad T, Pinto AF, Solbakk AK. Attentional control and subjective executive function in treatment-naive adults with Attention Deficit Hyperactivity Disorder. PLoS One. 2014;9(12):e115227.

74. Solsnes AE, Skranes J, Brubakk AM, Lohaugen GC. Executive functions in very-low-birth-weight young adults: a comparison between self-report and neuropsychological test results. $J$ Int Neuropsychol Soc. 2014;20(5):506-15.

75. Seltzer MM, Baker MW, Hong J, Maenner M, Greenberg J, Mandel D. Prevalence of CGG expansions of the FMR1 gene in a US population-based sample. Am J Med Genet B Neuropsychiatr Genet. 2012;159B(5):589-97.

76. Klusek J, Hong J, Sterling A, Berry-Kravis E, Mailick MR. Inhibition deficits are modulated by age and CGG repeat length in carriers of the FMR1 premutation allele who are mothers of children with fragile X syndrome. Brain Cogn. 2020;139:105511.

77. IBM Corp. IBM SPSS Statistics, Version 26.0. Armonk, NY: IBM Corp.; 2019.

78. Kim K, Hessl D, Randol JL, Espinal GM, Schneider A, Protic D, et al. Association between IQ and FMR1 protein (FMRP) across the spectrum of CGG repeat expansions. PLoS One. 2019;14(12):e0226811.

79. Hall D, Todorova-Koteva K, Pandya S, Bernard B, Ouyang B, Walsh M, et al. Neurological and endocrine phenotypes of fragile X carrier women. Clin Genet. 2016;89(1):60-7.

80. Kraan CM, Hocking DR, Georgiou-Karistianis N, Metcalfe SA, Archibald AD, Fielding J, et al. Impaired response inhibition is associated with self-reported symptoms of depression, anxiety, and ADHD in female FMR1 premutation carriers. Am J Med Genet B Neuropsychiatr Genet. 2014;165B(1):41-51.

\section{Tables}

Due to technological limitations, the tables can only be accessed as downloads in the supplementary files section.

\section{Figures}




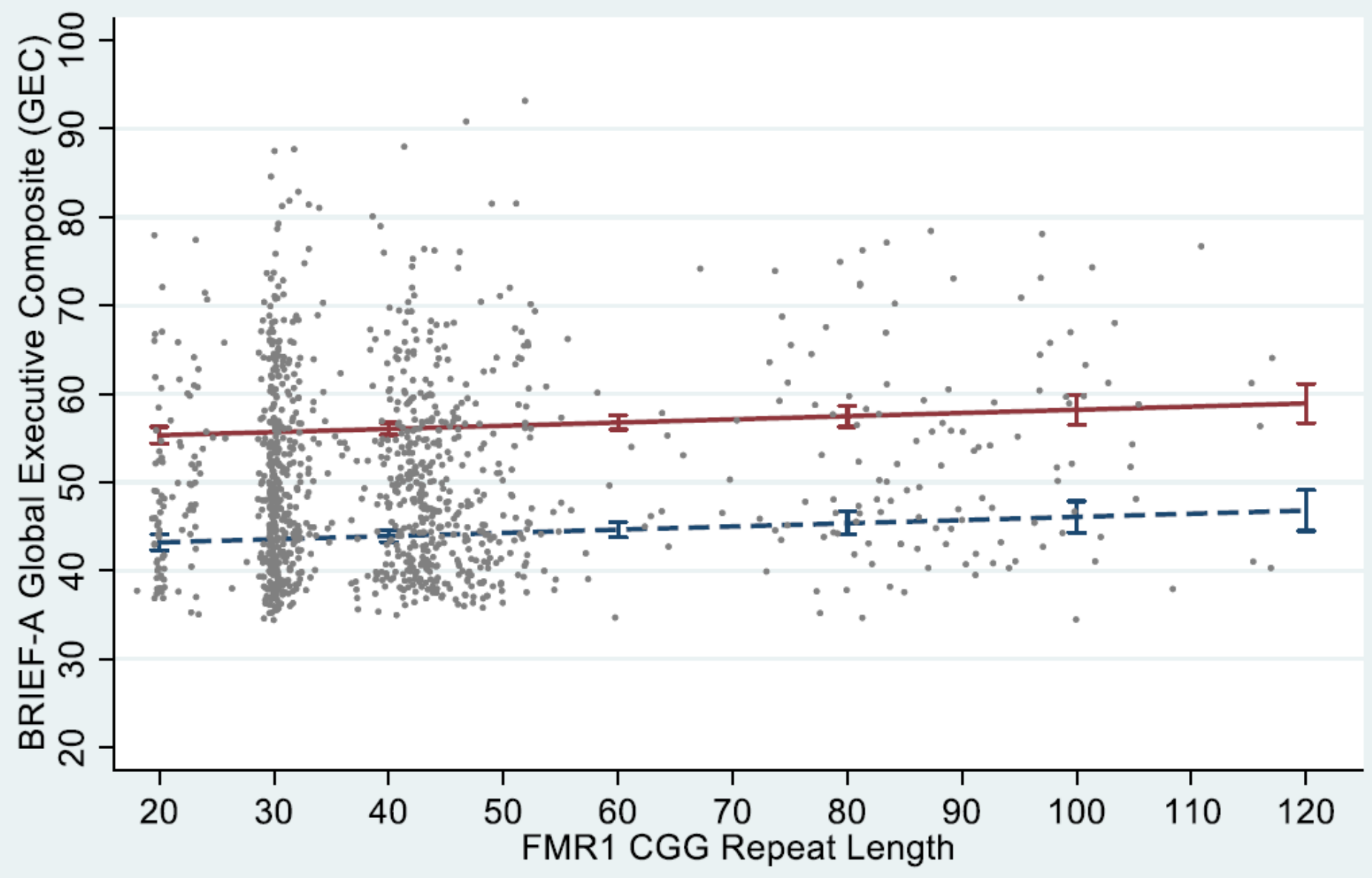

\section{Figure 1}

Perceived stress and CGG repeats predict GEC. Estimated regression lines indicate the independent influences of CGG repeat length and increased perceived stress (PSS) on executive function difficulty. Trend lines represent a subset of participants with high PSS (>1 SD above the mean) and low PSS scores ( $<1$ SD below the mean). 


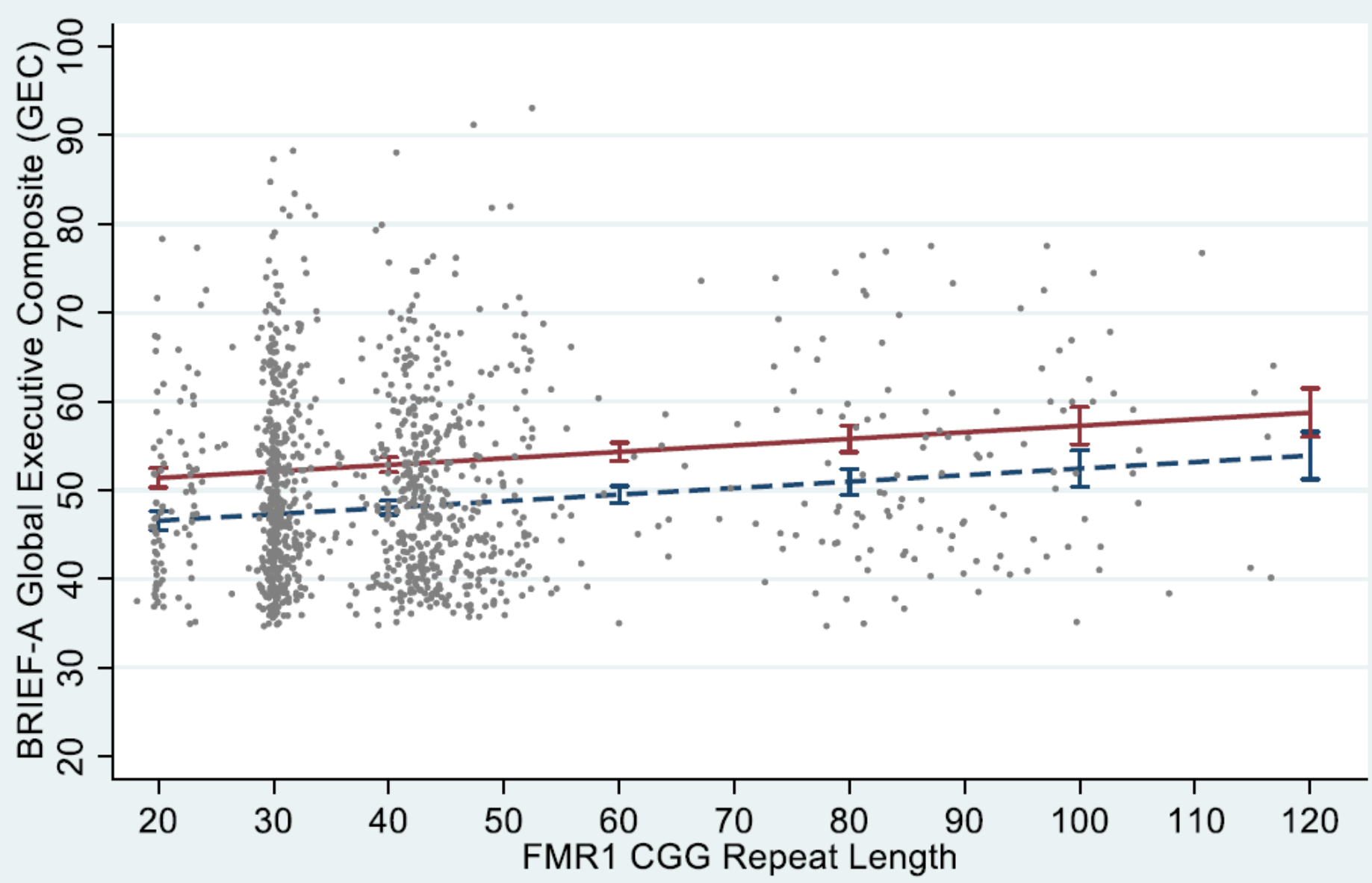

-ーーー- Low Life Events — High Life Events

\section{Figure 2}

Life events and CGG repeats predict GEC. Estimated regression lines indicate the independent influences of CGG repeat length and increased number of life events on executive function difficulty. Trend lines represent a subset of participants with high life events ( $>1$ SD above the mean) and low life events ( $<1$ SD below the mean). 


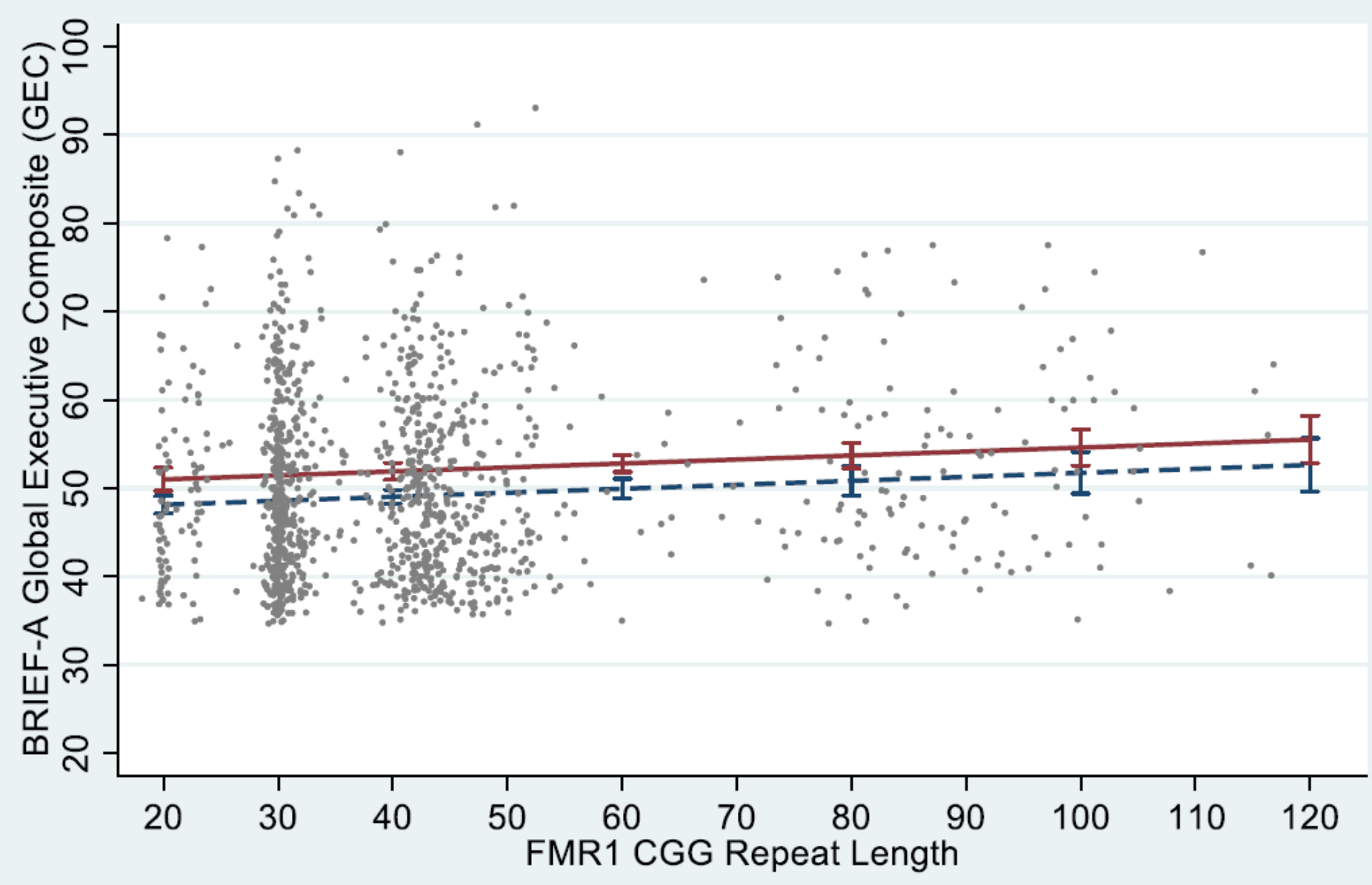

----- No Child with a Disability Child with a Disability

\section{Figure 3}

Parenting status and CGG repeats predict GEC. Estimated regression lines indicate the independent influences of CGG repeat length and parenting a child with a disability on executive function difficulty. 


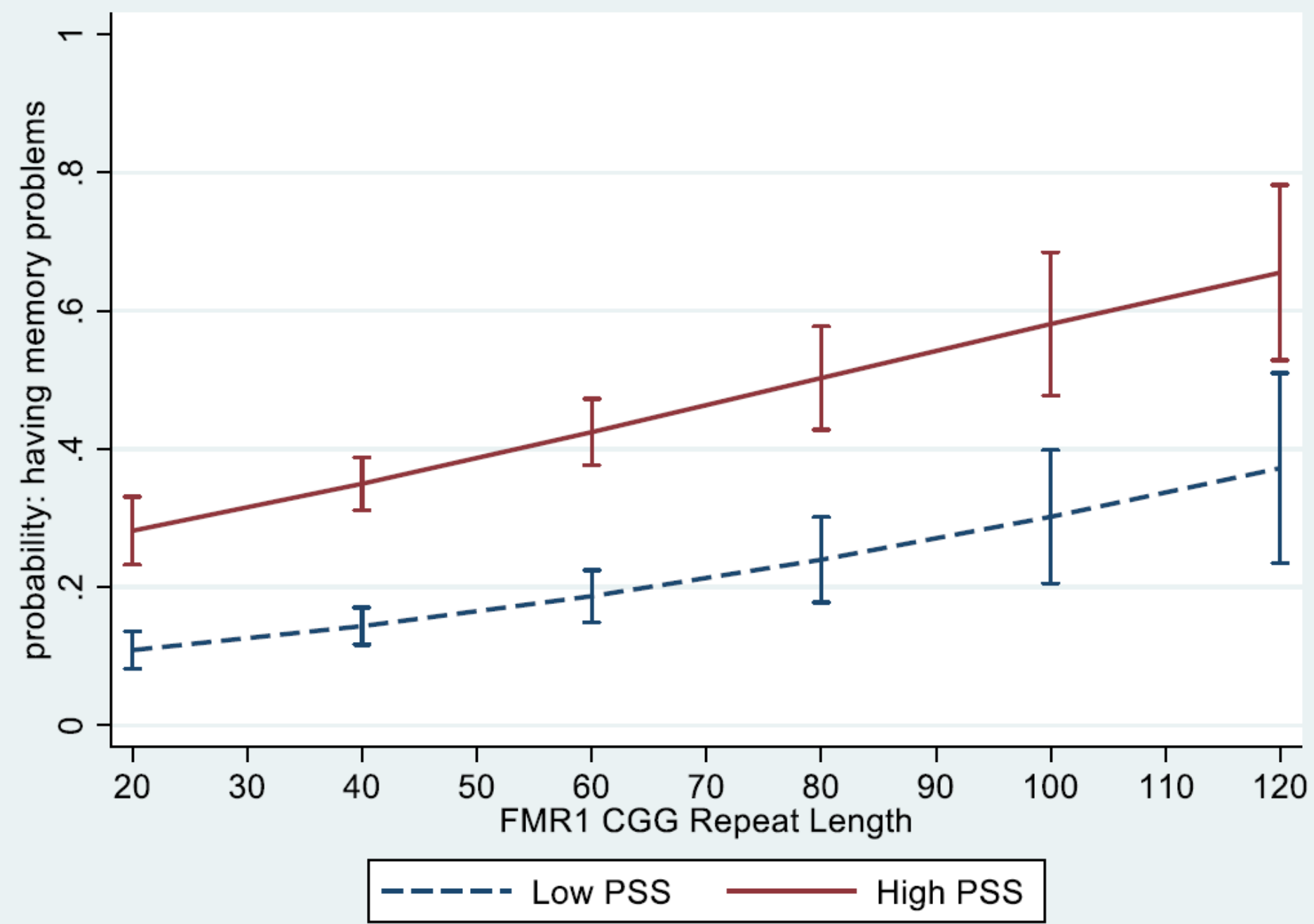

Figure 4

Linear association between CGG repeat length and the probability of self-reported memory problems. Trend lines represent estimated probability for participants with high PSS (>1 SD above the mean) and low PSS scores (<1 SD below the mean). 


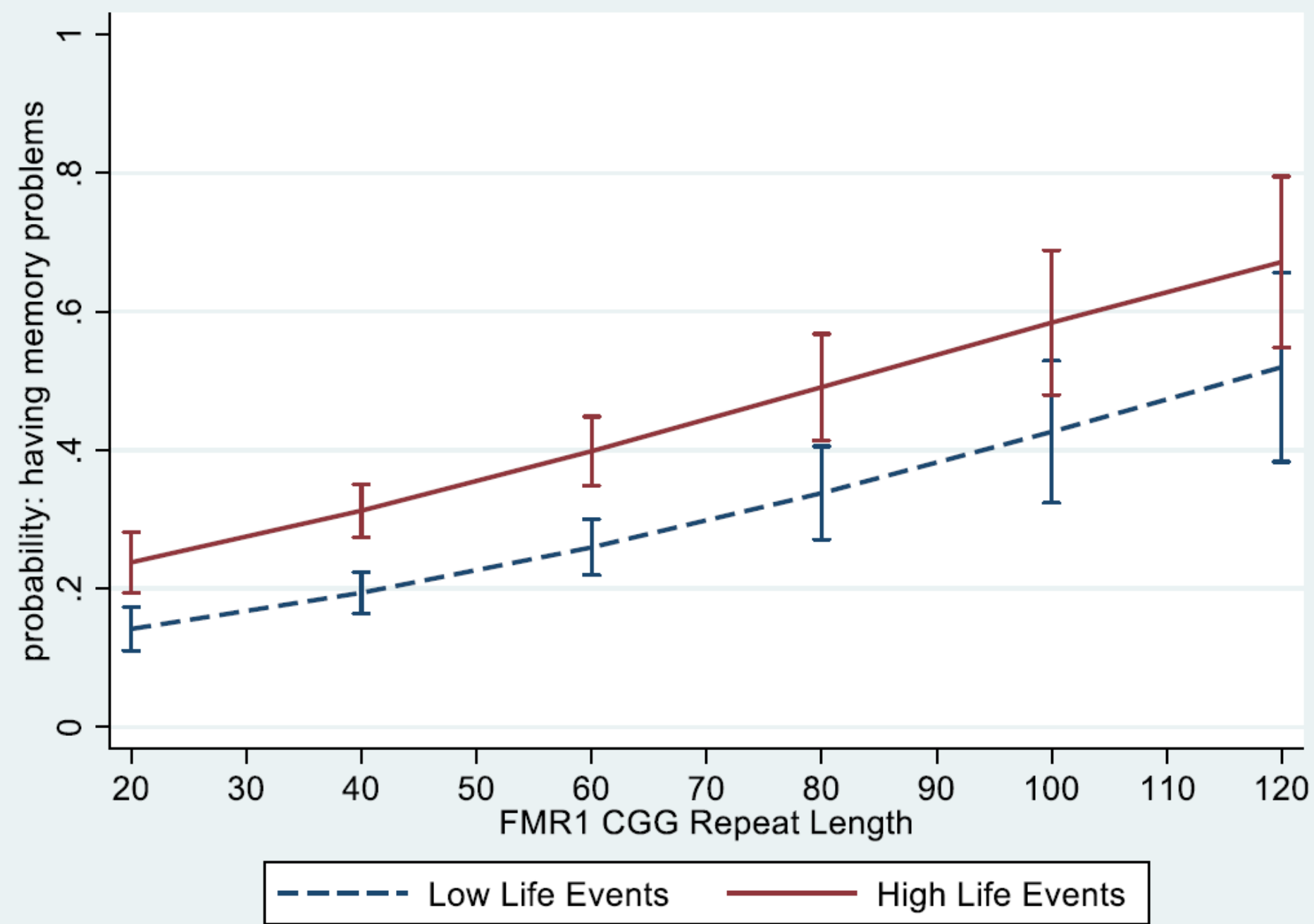

Figure 5

Linear association between CGG repeat length and the probability of self-reported memory problems. Trend lines represent estimated probability for participants with high life events (>1 SD above the mean) and low life events ( $<1$ SD below the mean). 


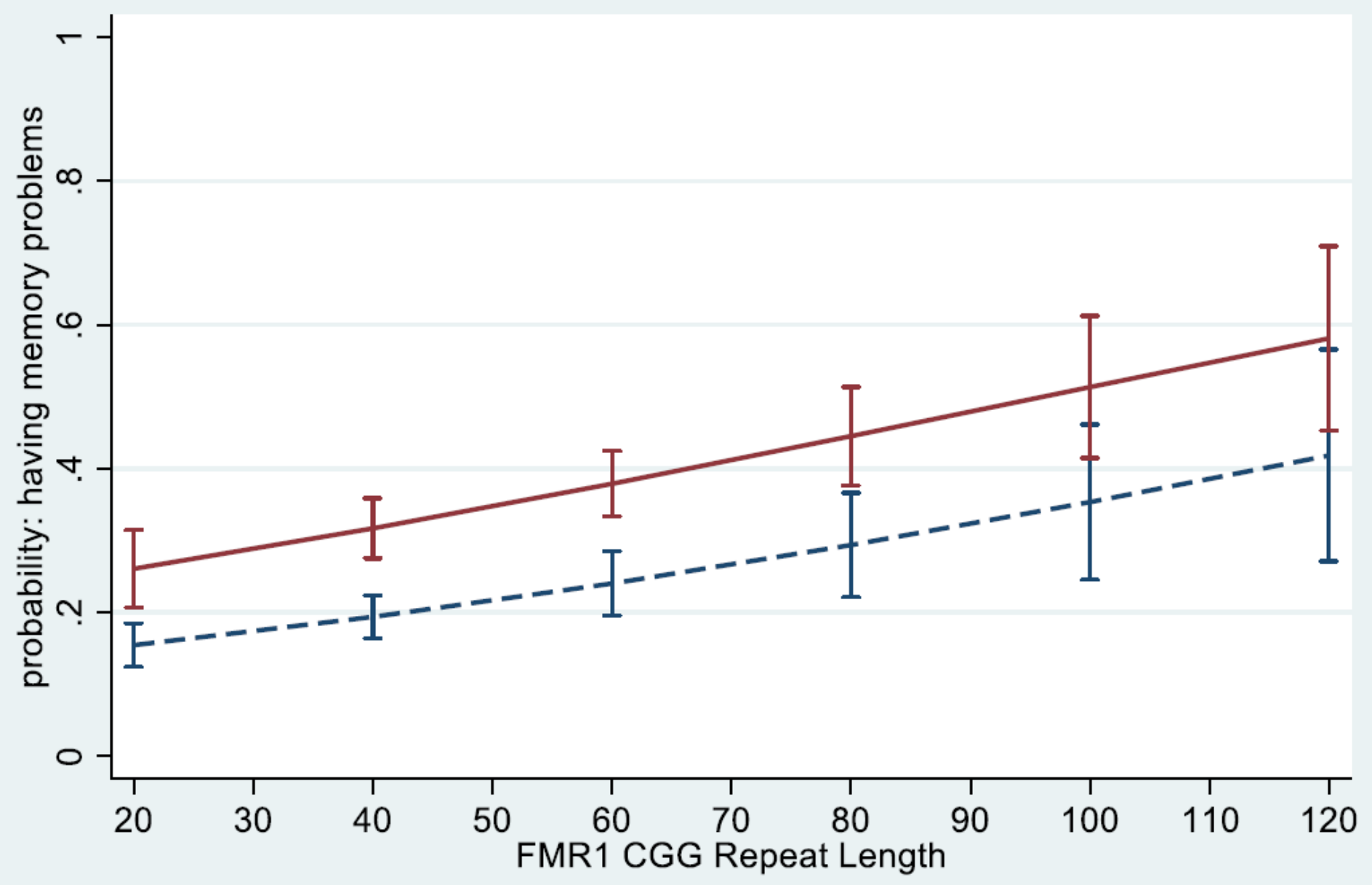

- - - - No Child with a Disability Child with a Disability

Figure 6

Linear association between CGG repeat length and the probability of self-reported memory problems by parenting status.

\section{Supplementary Files}

This is a list of supplementary files associated with this preprint. Click to download.

- Table1.pdf

- Table2.pdf

- Table3.pdf

- Table4.pdf

- Table7.Supplementary.pdf

- Table5.pdf

- Table6.Supplementary.pdf 